\title{
Conclusions:
}

The results of this audit highlight room for improvement in lithium monitoring of older adults attending POA service. Furthermore, all patients prescribed lithium had impaired renal function. This is an important finding given the associations between those admitted to hospital with COVID-19 and comorbid kidney disease and increased risk of inpatient death.

Our findings highlight the need for three monthly renal function monitoring in elderly prescribed lithiumgiven the additive adverse effects of increasing age and lithium on the kidney. Close working with specialised renal services to provide timely advice on renal management for those with renal impairment prescribed lithium is important to minimise adverse patient outcomes.

\section{3 - Episodes of lucidity (paradoxical lucidity): A survey of family caregivers of persons with dementia}

Presenter: Joan M. Griffin, Ph.D.

\section{Co-authors:}

Kyungmin Kim, PhD

Theresa Frangiosa, MBA

Virginia S. Biggar

Dawn M. Finnie, M.P.A.

Lauren Bangerter, PhD

Joseph E. Gaugler, PhD

Maria I. Lapid, MD

Keywords: advanced dementia, end of life, lucid intervals, caregivers

Topic: Dementia - Others

\begin{abstract}
:
People with late-stage Alzheimer's disease and related dementias (ADRD) who are assumed to have lost coherent cognitive capacity may exhibit unexpected episodes of spontaneous, meaningful, and relevant communication or behavior. Most reports of paradoxical lucidity or "episodes of lucidity" (EL) are anecdotal or case studies. Given the transient nature and lack of scientific explanation of the phenomenon, EL is under-investigated and poorly understood.
\end{abstract}

To develop an operational definition of and typologies for EL, we conducted a pilot study of former and current family caregivers from UsAgainstAlzheimer's A-LIST ${ }^{\circledR}(N=480)$. Over sixty percent of caregivers $(n=294,61 \%)$ reported witnessing at least one EL with their care recipient over the course of dementia. Most episodes happened in late stages of dementia (71\%). Only $10 \%$ happened within 7 days before death. The majority of episodes $(71 \%)$ lasted $<30$ minutes. About half the episodes were characterized by uncharacteristic speech and communication. Caregivers perceived these experiences positively $(M=$ 4.1 ; range $=1-5$ ), but also expressed desire to know why/when EL occurs and how to respond to it.

We plan to use these data to refine definitions and typologies to incorporate into a prospective, demographically diverse survey to family caregivers to assess predictors of EL and linking EL to caregiver well-being and bereavement response. Precise and robust operationalizations of EL will allow future research to assess if EL has different effects on ADRD prognosis or alters how family members understand, manage and adapt to a PLWD's dementia progression. 